\title{
Embedded gateway services for Internet of Things applications in ubiquitous healthcare
}

\begin{abstract}
The continuous advancement in computer and communication technologies has made personalized healthcare monitoring a rapidly growing area of interest. New features and services are envisaged, raising users' expectations in healthcare services. The emergence of Internet of Things brings people closer to connect the physical world to the Internet. In this paper, we present embedded services that are part of a ubiquitous healthcare system that allows automated and intelligent monitoring. The system uses IP connectivity and the Internet for end-to-end communication, from each 6LoWPAN sensor nodes to the web user interface on the Internet. The proposed algorithm in the Gateway performs multithreaded processing on the gathered medical signals for conversion to real data, feature extraction and wireless display. The user interface at the server allows users to access and view the medical data from mobile and portable devices. The ubiquitous system is exploring possibilities in connecting Internet with things and people for health services.
\end{abstract}

Keyword: 6LoWPAN; Embedded system; Internet of Things; Medical sensor networks; Ubiquitous health 